\title{
Superintegrability in a two-dimensional space of nonconstant curvature
}

\author{
E. G. Kalnins ${ }^{\text {a) }}$ and J. M. Kress ${ }^{\text {b) }}$ \\ Department of Mathematics, University of Waikato, Private Bag 3105, Hamilton, \\ New Zealand \\ P. Winternitz ${ }^{\mathrm{c}}$ \\ Centre de recherches mathématiques, Université de Montréal, C.P. 6128-CV, Montréal, \\ Québec H3C 3J7, Canada
}

(Received 21 August 2001; accepted for publication 1 November 2001)

\begin{abstract}
A Hamiltonian with two degrees of freedom is said to be superintegrable if it admits three functionally independent integrals of the motion. This property has been extensively studied in the case of two-dimensional spaces of constant (possibly zero) curvature when all the independent integrals are either quadratic or linear in the canonical momenta. In this article the first steps are taken to solve the problem of superintegrability of this type on an arbitrary curved manifold in two dimensions. This is done by examining in detail one of the spaces of revolution found by G. Koenigs. We determine that there are essentially three distinct potentials which when added to the free Hamiltonian of this space have this type of superintegrability. Separation of variables for the associated Hamilton-Jacobi and Schrödinger equations is discussed. The classical and quantum quadratic algebras associated with each of these potentials are determined. (C) 2002 American Institute of Physics. [DOI: 10.1063/1.1429322]
\end{abstract}

\section{INTRODUCTION}

A Hamiltonian system in classical mechanics with $n$ degrees of freedom is described by a Hamiltonian function $H\left(x_{1}, \ldots, x_{n}, p_{1}, \ldots, p_{n}\right)=H(x, p)$. The dynamics of such a system is described by Hamilton's equations

$$
\dot{x}_{i}=\frac{\partial H}{\partial p_{i}}, \quad \dot{p}_{i}=-\frac{\partial H}{\partial x_{i}} .
$$

The time rate of change of a classical observable $\ell=\ell(x, p)$ is given by

$$
\frac{d \ell}{d t}=\{\ell, H\}=\sum_{i=1}^{n}\left(\frac{\partial \ell}{\partial x_{i}} \frac{\partial H}{\partial p_{i}}-\frac{\partial \ell}{\partial p_{i}} \frac{\partial H}{\partial x_{i}}\right),
$$

where $\{$,$\} is the Poisson bracket. A Hamiltonian system is called "Liouville integrable" if it admits$ $n$ functionally independent integrals of motion $\left\{X_{1}, \ldots, X_{n}\right\}$ which are mutually in involution, i.e.,

$$
\left\{X_{i}, X_{j}\right\}=0, \quad i, j=1, \ldots, n,
$$

where one of these constants can be taken to be the Hamiltonian $H .{ }^{1,2}$ The system is superintegrable if a further $m$ integrals $\left\{Y_{1}, \ldots, Y_{m}, 1 \leqslant m \leqslant n-1\right\}$ exist such that the set of constants $\left\{X_{1}\right.$ $\left.=H, X_{2}, \ldots, X_{n}, Y_{1}, \ldots, Y_{m}\right\}$ is functionally independent. The additional integrals have vanishing

\footnotetext{
${ }^{a)}$ Electronic mail: e.kalnins@waikato.ac.nz

b)Electronic mail: jonathan@math.waikato.ac.nz

${ }^{c}$ Electronic mail: wintern@crm.umontreal.ca
} 
Poisson bracket with $H$, but not necessarily with each other or with the $X_{i}$ 's. A classical Hamiltonian system is maximally superintegrable if $m=n-1$. There are then $2 n-1$ functionally independent integrals of motion. The concepts of complete integrability and superintegrability have their analog in quantum mechanics. In this case a superintegrable quantum mechanical system is described by $n+m$ quantum observables $\left\{\hat{X}_{1}=\hat{H}, \hat{X}_{2}, \ldots, \hat{X}_{n}, \hat{Y}_{1}, \ldots, \hat{Y}_{m}\right\}$ which satisfy the commutation relations

$$
\left[\hat{H}, \hat{X}_{i}\right]=\hat{H} \hat{X}_{i}-\hat{X}_{i} \hat{H}=0, \quad\left[\hat{H}, \hat{Y}_{j}\right]=0, \quad\left[\hat{X}_{i}, \hat{X}_{k}\right]=0,
$$

where $i, k=1, \ldots, n, j=1, \ldots, m$. For superintegrable classical Hamiltonian systems it is often the case that the elements of our set of constants are polynomial in the canonical momenta. The best known maximally superintegrable systems in Euclidean space $E_{n}$ are the Kepler problem and the harmonic oscillator. All finite (bounded) trajectories in these two systems are closed. Moreover, these are the only spherically symmetric potentials for which all finite trajectories are closed. ${ }^{3}$

Systematic studies of superintegrable systems have been conducted for spaces of constant curvature in two and three dimensions. ${ }^{4-10}$ In particular, a complete classification of all superintegrable systems in the real Euclidean spaces $E_{2}$ and $E_{3}$ with at most second order integrals of motion was given. ${ }^{4-8}$ More recently, a relation between superintegrable systems and generalized Lie symmetries has been established, ${ }^{11}$ as well as their relation to exactly solvable problems in quantum mechanics. ${ }^{12}$ Recently ${ }^{13-15}$ it has been possible to classify all maximally superintegrable systems for spaces of constant curvature (possibly zero) in two dimensions for which all the extra constants of the motion are at most quadratic in the canonical momenta.

A natural question to ask is whether the concept of superintegrability is restricted to spaces of constant curvature. The purpose of this article is to show that this is not so and to start a study of superintegrable systems in more general Riemannian, pseudo-Riemannian and complex Riemannian spaces. More specifically, we consider real two-dimensional spaces and search for Hamiltonian systems allowing additional constants of the motion that are at most quadratic in the momenta.

To make initial progress on this problem we first need to know which Riemannian spaces in two dimensions have associated with them more than one classical quadratic constant of the motion. This is a problem that has been comprehensively solved by Koenigs ${ }^{16}$ in a note written in Vol. IV of the treatise of Darboux..$^{17}$

In addition to being of intrinsic interest, additional motivation for this problem comes from the observation that all two-dimensional Riemannian spaces can be embedded in the threedimensional Euclidean or pseudo-Euclidean space. Consequently, any such two-dimensional classical motion is equivalent to a constrained motion in three dimensions. It is also possible to interpret the motion, via general relativity, as motion in a two-dimensional gravitational field.

Given that we have a Riemannian space in two dimensions with infinitesimal distance

$$
d s^{2}=g_{i j}(u) d u^{i} d u^{j}, \quad i, j=1,2,
$$

and $u=\left(u^{1}, u^{2}\right)$, the classical Hamiltonian has the form

$$
H=\frac{1}{2} g^{i j} p_{i} p_{j}+V(u)
$$

and the corresponding Schrödinger equation can be taken to have the form

$$
\hat{H} \Psi=-\frac{1}{2 \sqrt{g}} \partial_{u^{i}}\left(\sqrt{g} g^{i k} \partial_{u^{k}} \Psi\right)+V(u) \Psi=E \Psi,
$$

where $g=\operatorname{det}\left(g_{i j}\right)$. For the classical Hamiltonian $H$ our problem is to look for potentials $V(u)$ and Riemannian spaces specified by the metric $g_{i j}$ for which there are at least two extra functionally independent constants of the motion of the form

$$
\lambda_{1}=a^{i j}(u) p_{i} p_{j}+b(u)
$$




$$
\lambda_{2}=a^{i}(u) p_{i}+c(u),
$$

other than $H$. One well known way of solving the corresponding classical problem is to use Hamilton-Jacobi theory. The crucial equation to solve is then the Hamilton-Jacobi equation obtained from the equation $H=E$ via the substitution $p_{i}=\partial S / \partial u^{i}$, that is,

$$
H=\frac{1}{2} g^{i j} \frac{\partial S}{\partial u^{i}} \frac{\partial S}{\partial u^{j}}+V(u)=E .
$$

This equation is sometimes solvable by the method of separation of variables using the additive separation ansatz

$$
S=S_{1}\left(u^{1}, \alpha, E\right)+S_{2}\left(u^{2}, \alpha, E\right) .
$$

The corresponding Schrödinger equation can also be solved by separation of variables with the product ansatz

$$
\Psi=\psi_{1}\left(u^{1}, \lambda, E\right) \psi_{2}\left(u^{2}, \lambda, E\right) .
$$

The quantities $\lambda_{i}$ are constants of the motion if

$$
\left\{\lambda_{i}, H\right\}=0 .
$$

For $\lambda_{2}$ this implies that $a^{i}(u)$ is a Killing vector and $a^{i}(u) p_{i}$ is a symmetry of the free Hamiltonian $[H$ without $V(u)]$. In the case of $\lambda_{1}$ this implies that $a^{i j}(u)$ is a Killing tensor. Such tensors are directly related to the notion of additive separation as described above. We note that for constants of the type $\lambda_{2}$, the condition implies $c(u)=0$. It is also clear that for every constant linear in the momenta, its square is a constant quadratic in the momenta, that is, of the form of $\lambda_{1}$.

As mentioned earlier, Darboux and Koenigs have given a comprehensive analysis of when a two-dimensional Riemannian space admits more than one quadratic constant. In Sec. II we summarize some of these results. ${ }^{16,17}$ In the remaining sections we concentrate on a particular space with a Killing vector and two Killing tensors. Section III deals with the free Hamilton-Jacobi equation and we show that the Schrödinger equation allows separation of variables in three different coordinates systems which we determine explicitly. Potentials that allow separation of variables in these systems are then introduced. In Sec. IV we find all potentials with this superintegrability property. We then discuss in Sec. V the various surfaces that may be represented by the infinitesimal distances that we have and the consequent special functions that arise from the corresponding Schrödinger equation.

\section{ON GEODESICS WITH QUADRATIC INTEGRALS}

In 1889 G. Koenigs ${ }^{16}$ wrote a note in the last volume of Darboux's treatise "Théorie générale de surfaces," the title of which coincides with the title of this section. This note contains a summary of results which are the solution of the problem outlined in the Introduction, viz. when does the free Hamiltonian of a two-dimensional Riemannian space admit more than one quadratic constant of the motion. The analysis was performed over the field of complex numbers and must be modified over the reals. What Koenigs did was to write the infinitesimal distance for a general two-dimensional Riemannian space in the form

$$
d s^{2}=4 f(x, y) d x d y .
$$

This can always be done in two dimensions over C. The corresponding free Hamiltonian then has the form 


$$
H=\frac{1}{2 f(x, y)} p_{x} p_{y} .
$$

By making the requirement that there is a second order Killing tensor of the form

$$
\lambda=a^{i j}(u) p_{i} p_{j}
$$

Darboux and Koenigs establish the following propositions.

(1) Any two-dimensional Riemannian space that admits more than one Killing vector must be a space of constant curvature and admit three linearly independent Killing vectors.

(2) Any two-dimensional Riemannian space that admits more than three Killing tensors is a space of constant curvature. It then actually admits five linearly independent Killing tensors which are all bilinear expressions in the Killing vectors. The sixth bilinear combination is the Hamiltonian itself.

(3) Any two-dimensional Riemannian space that admits precisely three linearly independent Killing tensors will be a Riemannian space of revolution. In fact, there will be one Killing vector and two Killing tensors.

Two-dimensional Riemannian spaces of this latter type were distinguished to be of four types. The infinitesimal distances of these types are given by

(I) $d s^{2}=(x+y) d x d y$,

(II) $d s^{2}=\left(a /(x-y)^{2}+b\right) d x d y$,

(III) $d s^{2}=\left(a e^{-(x+y) / 2}+b e^{-x-y}\right) d x d y$,

(IV) $d s^{2}=\left(a\left(e^{(x-y) / 2}+e^{(y-x) / 2}\right)+b\right) /\left(e^{(x-y) / 2}-e^{(y-x) / 2}\right)^{2} d x d y$.

It is the first of these infinitesimal distances that we analyze in some detail in the next section. We shall call the spaces "Darboux spaces" and denote them by $D_{1}, D_{2}, D_{3}$ and $D_{4}$, respectively.

\section{THE FREE PARTICLE AND SEPARATION OF VARIABLES IN A DARBOUX SPACE OF TYPE ONE}

If we consider the first space of Darboux's list and look at real forms of this space only, it is convenient to make the new choice of variables

$$
x=u+i v, \quad y=u-i v .
$$

The corresponding infinitesimal distance can then be taken as

$$
d s^{2}=2 u\left(d u^{2}+d v^{2}\right)
$$

and the corresponding Hamiltonian has the form

$$
H=\frac{1}{4 u}\left(p_{u}^{2}+p_{v}^{2}\right) .
$$

Associated with this Hamiltonian are three integrals of the free motion, two quadratic and one linear:

$$
\begin{aligned}
& K=p_{v}, \quad X_{1}=p_{u} p_{v}-\frac{v}{2 u}\left(p_{u}^{2}+p_{v}^{2}\right), \\
& X_{2}=p_{v}\left(v p_{u}-u p_{v}\right)-\frac{v^{2}}{4 u}\left(p_{u}^{2}+p_{v}^{2}\right) .
\end{aligned}
$$

These three integrals satisfy the polynomial Poisson algebra relations, 


$$
\left\{K, X_{1}\right\}=2 H, \quad\left\{K, X_{2}\right\}=-X_{1}, \quad\left\{X_{1}, X_{2}\right\}=2 K^{3} .
$$

They cannot be functionally independent and, in fact, satisfy the relation

$$
4 H X_{2}+X_{1}^{2}+K^{4}=0 .
$$

For the analogous quantum problem it is sufficient to consider the operators

$$
\begin{gathered}
\hat{H}=-\frac{1}{4 u}\left(\partial_{u}^{2}+\partial_{v}^{2}\right), \quad \hat{K}=-i \partial_{v}, \\
\hat{X}_{1}=-\partial_{u} \partial_{v}+\frac{v}{2 u}\left(\partial_{u}^{2}+\partial_{v}^{2}\right), \quad \hat{X}_{2}=-\frac{1}{2}\left[\partial_{v}, v \partial_{u}-u \partial_{v}\right]_{+}+\frac{v^{2}}{4 u}\left(\partial_{u}^{2}+\partial_{v}^{2}\right),
\end{gathered}
$$

where $[A, B]_{+}=A B+B A$. The quantum versions of the quadratic constants are obtained via the formula

$$
\hat{\lambda}=-\frac{1}{\sqrt{g}} \partial_{i}\left(a^{i j} \sqrt{g} \partial_{j}\right) .
$$

These operators have the same commutation relations as for the classical constants with the Poisson bracket replaced by the commutator bracket:

$$
\left[\hat{K}, \hat{X}_{1}\right]=2 i \hat{H}, \quad\left[\hat{K}, \hat{X}_{2}\right]=-i \hat{X}_{1}, \quad\left[\hat{X}_{1}, \hat{X}_{2}\right]=-2 i \hat{K}^{3} .
$$

There is also the operator relation

$$
4 \hat{H} \hat{X}_{2}+\hat{X}_{1}^{2}+\hat{K}^{4}=0 .
$$

The question we address in this section relates to the various possible ways that separation of variables can be achieved in the case of free classical motion or its quantum analogue, the free Schrödinger equation. The criteria for this to occur is the same in either case. Classically, if we have a general quadratic first integral $\lambda$ and free Hamiltonian

$$
H=\frac{1}{2} g^{i j}(u) p_{i} p_{j},
$$

and if the characteristic equation,

$$
\left|a^{i j}-\rho g^{i j}\right|=0,
$$

has two distinct roots $\rho_{1}$ and $\rho_{2}$, the Hamiltonian will have Liouville form when written in terms of the new variables $\rho_{1}, \rho_{2}$. That is,

$$
H=\frac{\sigma\left(\rho_{1}\right) p_{\rho_{1}}^{2}+\tau\left(\rho_{2}\right) p_{\rho_{2}}^{2}}{\rho_{1}+\rho_{2}} .
$$

In this form, both classical and quantum systems can be solved by the separation of variables ansatz.

If we want to classify all different separable coordinate systems for a given Hamiltonian, we need to know how many essentially different quadratic first integrals are possible. To decide on the notion of equivalence we first observe that the variable $v$ does not explicitly appear in the metric tensor, that is, it is an ignorable variable. This means that the transformations $v \rightarrow v+b$ form a 
one-dimensional Lie group. Accordingly, we determine the notion of equivalence to mean that two quadratic integrals are equivalent if they are related by a motion of this group. Consequently, the most general quadratic constant can be written

$$
\lambda=a X_{1}+b X_{2}+c K^{2}
$$

to within the addition of a multiple of $H$. The second order elements $X_{i}$ transform under the adjoint action according to

$$
X_{i} \rightarrow \exp (\alpha K) X_{i} \exp (-\alpha K)=\exp (\alpha A d(K)) X_{i}=X_{i}+\alpha\left\{K, X_{i}\right\}+\frac{1}{2} \alpha^{2}\left\{K,\left\{K, X_{i}\right\}\right\}+\ldots,
$$

or specifically

$$
X_{1} \rightarrow X_{1}+2 \alpha H, \quad X_{2} \rightarrow X_{2}-\alpha X_{1}-\alpha^{2} H .
$$

There are three classes of possible quadratic first integrals under this equivalence relation. Typical representatives are

$$
X_{1}+a K^{2}, \quad X_{2}+a K^{2}, \quad K^{2} .
$$

We can now explicitly demonstrate the separable coordinates in each of these cases.

(1) Separating coordinates associated with $X_{1}+a K^{2}$. If we choose a representative to be

$$
L=X_{1}+\sinh c K^{2},
$$

the corresponding roots of the characteristic equation and hence new variables are

$$
r=\rho_{1}=-2(C u+v), \quad s=\rho_{2}=\frac{2}{C}(u-C v), \quad C=e^{-c} .
$$

In terms of these coordinates the Hamiltonian has the form

$$
H=\frac{2\left(C^{2}+1\right)^{2}}{C(s-r)}\left(\frac{1}{C^{2}} p_{s}^{2}+p_{r}^{2}\right),
$$

and the corresponding quadratic constant in these coordinates is

$$
L=2 \frac{\left(C^{2}+1\right)^{2}}{C(s-r)}\left(\frac{r}{C^{2}} p_{s}^{2}+s p_{r}^{2}\right) .
$$

(2) Separating coordinates associated with $X_{2}+a K^{2}$. Taking the second representative in the list (33), that is, $L=X_{2}+a K^{2}$, a convenient choice of new variables $\xi, \eta$ is related to the roots $\rho_{i}$ by

$$
\rho_{1}=\eta^{2}\left(2 a-\eta^{2}\right), \quad \rho_{2}=-\xi^{2}\left(2 a+\xi^{2}\right) .
$$

The corresponding classical Hamiltonian then has the form

$$
H=\frac{p_{\xi}^{2}+p_{\eta}^{2}}{2\left(\xi^{2}+\eta^{2}\right)\left(\xi^{2}-\eta^{2}+2 a\right)} .
$$

The associated constant of the motion in the new coordinates $\xi$ and $\eta$ is

$$
L=\frac{\eta^{2}\left(2 a-\eta^{2}\right) p_{\xi}^{2}-\xi^{2}\left(\xi^{2}+2 a\right) p_{\eta}^{2}}{2\left(\xi^{2}+\eta^{2}\right)\left(\xi^{2}-\eta^{2}+2 a\right)}
$$


The defining coordinates $u, v$ are written in terms of the new coordinates $\xi, \eta$ via

$$
u=\frac{1}{2}\left(\xi^{2}-\eta^{2}\right)+a, \quad v=\xi \eta,
$$

which looks like displaced parabolic coordinates in the $u, v$ plane.

(3) Separating coordinates associated with $K^{2}$. For the last representative, $K^{2}$, we need only the coordinates $u, v$ and to recognize the fact that $K=p_{v}$.

We conclude this section by discussing the solutions to the free particle and free Schrödinger equation in these three cases.

In case 1 above it is more convenient to choose the variables according to

$$
u=r \cos \theta+s \sin \theta, \quad v=-r \sin \theta+s \cos \theta .
$$

The classical Hamilton-Jacobi equation then has the form

$$
H=\frac{(\partial S / \partial r)^{2}+(\partial S / \partial s)^{2}}{4(r \cos \theta+s \sin \theta)}=E
$$

which has the general separable solution

$$
S=S_{1}(r)+S_{2}(s)=\frac{(4 E r \cos \theta-\lambda)^{3 / 2}}{6 E \cos \theta}+\frac{(4 E s \sin \theta+\lambda)^{3 / 2}}{6 E \sin \theta} .
$$

The corresponding free Schrödinger equation

$$
\hat{H} \Psi=-\frac{1}{4(r \cos \theta+s \sin \theta)}\left(\partial_{r}^{2}+\partial_{s}^{2}\right) \Psi=E \Psi
$$

has the typical product solutions

$$
\begin{aligned}
\Psi= & \sqrt{\left(r-\frac{\mu}{4 E \cos \theta}\right)\left(s+\frac{\mu}{4 E \sin \theta}\right)} C_{1 / 3}\left(\frac{2}{3} \sqrt{4 E \cos \theta}\left(r-\frac{\mu}{4 E \cos \theta}\right)^{3 / 2}\right) \\
& \times C_{1 / 3}\left(\frac{2}{3} \sqrt{4 E \sin \theta}\left(s+\frac{\mu}{4 E \sin \theta}\right)^{3 / 2}\right),
\end{aligned}
$$

where $C_{\nu}(z)$ is a solution of Bessel's equation.

In the second case the classical Hamilton-Jacobi equation is

$$
H=\frac{(\partial S / \partial \xi)^{2}+(\partial S / \partial \eta)^{2}}{2\left(\xi^{2}+\eta^{2}\right)\left(\xi^{2}-\eta^{2}+2 c\right)}=E
$$

and has a general solution of the form

$$
S=\int \sqrt{2 E \xi^{4}+2 E c \xi^{2}-\lambda} d \xi+\int \sqrt{-2 E \eta^{4}+2 E c \eta^{2}+\lambda} d \eta,
$$

which can be expressed in terms of elliptic integrals. The corresponding Schrödinger equation has a solution of the form $\Psi=\psi_{1}(\xi) \psi_{2}(\eta)$, where the $\psi_{i}$ satisfy

$$
\begin{gathered}
\left(\partial_{\xi}^{2}+2 E \xi^{4}+4 E c \xi^{2}+\lambda\right) \psi_{1}(\xi)=0, \\
\left(\partial_{\eta}^{2}-2 E \eta^{4}+4 E c \eta^{2}-\lambda\right) \psi_{2}(\eta)=0 .
\end{gathered}
$$

These equations are readily identified as the equations for the anharmonic oscillator. 
In the third case the classical Hamilton-Jacobi equation is

$$
H=\frac{1}{4 u}\left(\left(\frac{\partial S}{\partial u}\right)^{2}+\left(\frac{\partial S}{\partial v}\right)^{2}\right)=E,
$$

which has separable solutions

$$
S=\frac{1}{6 E}\left(4 E u-k^{2}\right)^{3 / 2}+k v .
$$

The separable solutions to the corresponding free Schrödinger equation

$$
-\frac{1}{4 u}\left(\partial_{u}^{2}+\partial_{v}^{2}\right) \Psi=E \Psi
$$

have the form

$$
\Psi=\sqrt{u-\frac{m^{2}}{4 E}} C_{1 / 3}\left(\frac{2}{3} \sqrt{4 E}\left(u-\frac{m^{2}}{4 E}\right)^{3 / 2}\right) e^{i m v} .
$$

It is clear that the actual solutions to the classical motion or the corresponding Schrödinger equation depend on the range of values assumed by the various real variables, that is, on exactly which real manifold we are considering.

\section{INTEGRABLE AND SUPERINTEGRABLE SYSTEMS FOR THE DARBOUX SPACE OF TYPE ONE}

In this section we address the problem of superintegrability for the Hamiltonian

$$
H=\frac{1}{4 u}\left(p_{u}^{2}+p_{v}^{2}\right)
$$

that is, look for potentials $V(u, v)$ for which

$$
\bar{H}=H+V(u, v)
$$

admits at least two extra quadratic integrals. The way to solve this problem is as follows. First we consider that we already have one quadratic first integral

$$
\bar{L}=a(u, v) p_{u}^{2}+b(u, v) p_{u} p_{v}+c(u, v) p_{v}^{2}+d(u, v)
$$

We know that the quadratic part of $\bar{L}$ [i.e., that part obtained by putting $d(u, v)=0$ in (56)] must correspond to one of the three possibilities outlined in the previous section. For each of these possibilities separation of variables is possible in coordinates $\alpha, \beta$ where $u=u(\alpha, \beta), v$ $=v(\alpha, \beta)$. The addition of a potential implies that separation is preserved. As a consequence of this, $\bar{H}$ can be written as

$$
\bar{H}=\frac{p_{\alpha}^{2}+p_{\beta}^{2}+f(\alpha)+g(\beta)}{\sigma(\alpha)+\tau(\beta)}
$$

and the corresponding first integral will be

$$
\bar{L}=\frac{\sigma(\alpha)\left(p_{\beta}^{2}+g(\beta)\right)-\tau(\beta)\left(p_{\alpha}^{2}+f(\alpha)\right)}{\sigma(\alpha)+\tau(\beta)} .
$$


The next step is to impose the condition that there is a further quadratic first integral and see what conditions this imposes on the functions $f(\alpha)$ and $g(\beta)$. If we do these calculations systematically, we arrive at the following three cases.

(1)

$$
H=\frac{p_{u}^{2}+p_{v}^{2}}{4 u}+\frac{b_{1}\left(4 u^{2}+v^{2}\right)}{4 u}+\frac{b_{2}}{u}+\frac{b_{3}}{u v^{2}} .
$$

The additional constants of the motion have the form

$$
R_{1}=X_{2}-\frac{b_{1} v^{4}}{4 u}-\frac{b_{2} v^{2}}{u}-\frac{b_{3}\left(4 u^{2}+v^{2}\right)}{v^{2} u}, \quad R_{2}=K^{2}+b_{1} v^{2}+\frac{4 b_{3}}{v^{2}}
$$

and the corresponding quadratic algebra ${ }^{18,19}$ relations are determined by

$$
\begin{gathered}
\left\{R, R_{1}\right\}=8 H R_{1}+6 R_{2}^{2}+16 b_{2} R_{2}-32 b_{1} b_{3}, \\
\left\{R, R_{2}\right\}=-8 H R_{2}-16 b_{1} R_{1}, \\
R^{2}=-16 H R_{1} R_{2}-4 R_{2}^{3}-16 b_{2} R_{2}^{2}-64 b_{3} H^{2}-16 b_{1} R_{1}^{2}+64 b_{1} b_{3} R_{2}+256 b_{1} b_{2} b_{3},
\end{gathered}
$$

where $R=\left\{R_{1}, R_{2}\right\}$. The Hamiltonian clearly separates in the coordinates $u$ and $v$ as well as the coordinates $\xi, \eta$ given by $u=\frac{1}{2}\left(\xi^{2}-\eta^{2}\right)+a, v=\xi \eta$. This can be seen from the explicit form

$$
H=\frac{p_{\xi}^{2}+p_{\eta}^{2}}{2\left(\xi^{2}+\eta^{2}\right)\left(\xi^{2}-\eta^{2}+2 a\right)}+\frac{b_{1}\left(\left(\xi^{2}-\eta^{2}+2 a\right)^{2}+\xi^{2} \eta^{2}\right)+4 b_{2}+\left(4 b_{3} / \xi^{2} \eta^{2}\right)}{2\left(\xi^{2}-\eta^{2}+2 a\right)} .
$$

The corresponding quadratic quantum algebra relations are

$$
\begin{gathered}
{\left[\hat{R}, \hat{R}_{1}\right]=-6 \hat{R}_{2}^{2}-8 \hat{H} \hat{R}_{1}+16 b_{2} \hat{R}_{2}+2 b_{1}\left(3+16 b_{3}\right),} \\
{\left[\hat{R}^{,} \hat{R}_{2}\right]=8 \hat{H} \hat{R}_{2}-16 b_{1} \hat{R}_{1},} \\
\hat{R}^{2}=+4 \hat{R}_{2}^{3}-8 \hat{H}\left[\hat{R}_{1}, \hat{R}_{2}\right]_{+}-16 b_{2} \hat{R}_{2}^{2}-16 b_{1} \hat{R}_{1}^{2}-4 b_{1}\left(11+16 b_{3}\right) \hat{R}_{2} \\
-4\left(3+16 b_{3}\right) \hat{H}^{2}+16 b_{1} b_{2}\left(3+16 b_{3}\right),
\end{gathered}
$$

where $\hat{R}=\left[\hat{R}_{1}, \hat{R}_{2}\right]$.

(2)

$$
H=\frac{p_{u}^{2}+p_{v}^{2}}{4 u}+\frac{a_{1}}{u}+\frac{a_{2} v}{u}+\frac{a_{3}\left(u^{2}+v^{2}\right)}{u} .
$$

The additional constants of the motion have the form

$$
\begin{gathered}
R_{1}=X_{1}-\frac{2 a_{1} v}{u}+\frac{2 a_{2}\left(u^{2}-v^{2}\right)}{u}+\frac{2 a_{3} v\left(u^{2}-v^{2}\right)}{u}, \\
R_{2}=K^{2}+4 a_{2} v+4 a_{3} v^{2},
\end{gathered}
$$

and the corresponding quadratic algebra relations are determined by

$$
\left\{R, R_{1}\right\}=-8 H^{2}+16 a_{3} R_{2}+8\left(a_{2}^{2}+4 a_{1} a_{3}\right),
$$




$$
\begin{gathered}
\left\{R, R_{2}\right\}=16 a_{2} H-16 a_{3} R_{1}, \\
R^{2}=16 H^{2} R_{2}-16 a_{3} R_{2}^{2}+32 a_{2} H R_{1}-16 a_{3} R_{1}^{2}-16\left(a_{2}^{2}+4 a_{1} a_{3}\right) R_{2}-64 a_{1} a_{2}^{2} .
\end{gathered}
$$

If we change the coordinates according to $u=r \cos \theta+s \sin \theta, v=-r \sin \theta+s \cos \theta$, the Hamiltonian assumes the form

$$
H=\frac{p_{r}^{2}+p_{s}^{2}+4 a_{1}+4 a_{2}(-r \sin \theta+s \cos \theta)+4 a_{3}\left(r^{2}+s^{2}\right)}{4(r \cos \theta+s \sin \theta)},
$$

which clearly also separates in these coordinates.

The commutation relations of the corresponding quantum algebra are

$$
\begin{gathered}
{\left[\hat{R}, \hat{R}_{1}\right]=16 a_{3} \hat{R}_{2}+8 \hat{H}^{2}-8\left(a_{2}^{2}+4 a_{1} a_{3}\right),} \\
{\left[\hat{R}, \hat{R}_{2}\right]=-16 a_{3} \hat{R}_{1}+16 a_{2} \hat{H},} \\
\hat{R}^{2}=-16 a_{3} \hat{R}_{2}^{2}-16 a_{3} \hat{R}_{1}^{2}+16 \hat{H}^{2} \hat{R}_{2}+32 a_{2} \hat{H} \hat{R}_{1}-16\left(a_{2}^{2}+4 a_{1} a_{3}\right) \hat{R}_{2}+64\left(a_{3}^{2}-a_{1} a_{2}^{2}\right) .
\end{gathered}
$$

(3) The third potential gives rise to a Hamiltonian of the form

$$
H=\frac{p_{u}^{2}+p_{v}^{2}}{4 u}+\frac{a}{u}
$$

There are three extra constants associated with this Hamilonian,

$$
R_{1}=X_{1}-\frac{2 a v}{u}, \quad R_{2}=X_{2}-\frac{a v^{2}}{u} \text { and } K
$$

The associated Poisson bracket relations are

$$
\left\{K, R_{1}\right\}=2 H, \quad\left\{K, R_{2}\right\}=-R_{1}, \quad\left\{R_{1}, R_{2}\right\}=2 K\left(K^{2}+2 a\right),
$$

and the corresponding functional relation among these constants is

$$
4 H R_{2}+R_{1}^{2}+K^{4}+4 a K^{2}=0 .
$$

The commutation relations associated with the corresponding quantum problem have the form

$$
\left[\hat{K}, \hat{R}_{1}\right]=2 i \hat{H}, \quad\left[\hat{K}, \hat{R}_{2}\right]=-i \hat{R}_{1}, \quad\left[\hat{R}_{1}, \hat{R}_{2}\right]=-2 i \hat{K}\left(\hat{K}^{2}-2 a\right)
$$

and the identity amongst the defining operators is

$$
4 \hat{H} \hat{R}_{2}+\hat{R}_{1}^{2}+\hat{K}^{4}-4 a \hat{K}^{2}=0 .
$$

Upon examination of the various superintegrable potentials we have constructed, we see that by multiplying the equation $H=E$ by a suitable factor we essentially recover a variant of one of the superintegrable systems already classified for spaces of constant (or zero) curvature. For the first potential above, the equation $H=E$ may be written

$$
p_{u}^{2}+p_{v}^{2}+b_{1}\left(4 u^{2}+v^{2}\right)+4 b_{2}+\frac{4 b_{3}}{v^{2}}-4 E u=0
$$

This equation is known to have separable solutions in coordinates $u, v$ and associated parabolic coordinates $\xi, \eta$ given by $u=\frac{1}{2}\left(\xi^{2}-\eta^{2}\right), v=\xi \eta$. With the second potential, $H=E$ becomes 


$$
p_{u}^{2}+p_{v}^{2}+4 a_{3}\left(u^{2}+v^{2}\right)+4 a_{1}+4 a_{2} v-4 E u=0,
$$

and the third becomes

$$
p_{u}^{2}+p_{v}^{2}-4 E u+4 a=0 .
$$

This observation is crucial to the whole program that we will undertake which aims at finding all superintegrable systems associated with a curved space in two dimensions and having quadratic constants.

All three of the above systems are special cases of the superintegrable systems found in $E_{2} \cdot{ }^{4,11}$ They were shown to be exactly solvable in Ref. 12 .

\section{EMBEDDINGS OF A DARBOUX SPACE OF REVOLUTION OF TYPE ONE}

It is clear that the infinitesimal distance

$$
d s^{2}=2 u\left(d u^{2}+d v^{2}\right)
$$

does not uniquely determine a manifold. This then gives rise to the question of just what sort of surfaces can this infinitesimal distance represent. A particular choice of such a surface would determine the range of variation of the parameters $u, v$ which in turn enables the solution of the geodesic equations in the case of classical mechanics and the quantum mechanics of a point particle. It is known that any two-dimensional Riemannian space can be embedded in a threedimensional Euclidean space of indefinite or definite signature. In this section we look at a number of natural embeddings and discuss their associated geodesics and quantum mechanics. The infinitesimal distance that we are dealing with can be embedded in three-dimensional Euclidean space $E_{3}$ via the formulas

$$
\begin{aligned}
& X=\sqrt{2 u} \cos v, \quad Y=\sqrt{2 u} \sin v, \\
& Z=\frac{\sqrt{2}}{3}\left(F\left(\varphi, \frac{1}{\sqrt{2}}\right)+\sqrt{4 u^{3}-u}\right),
\end{aligned}
$$

where $u \geqslant \frac{1}{2}, v_{0} \leqslant v \leqslant 2 \pi+v_{0}, \sin \varphi=\sqrt{2 u+1}$ and $F(\varphi, k)$ is an elliptic integral of the first kind. This embedding gives the infinitesimal distance

$$
d X^{2}+d Y^{2}+d Z^{2}=2 u\left(d u^{2}+d v^{2}\right) .
$$

To do quantum mechanics on this surface let us first look for separable solutions to the free Schrödinger equation. A typical solution has already been found in the previous section, viz.

$$
\Psi=\sqrt{u-\frac{m^{2}}{4 E}} C_{1 / 3}\left(\frac{2}{3} \sqrt{4 E}\left(u-\frac{m^{2}}{4 E}\right)^{3 / 2}\right) e^{i m v},
$$

where $m$ is an integer. As $u \geqslant \frac{1}{2}$ and we see that $u=\frac{1}{2}$ is not a singular point of the separable equation in $u$, we can impose a condition of the form

$$
a \Psi\left(\frac{1}{2}, v\right)+b \Psi_{u}\left(\frac{1}{2}, v\right)=0
$$

together with the periodic boundary condition $\Psi(u, v)=\Psi(u, v+2 \pi)$, which is already satisfied. If we take $a=1, b=0$, then $E \geqslant 0$, otherwise there is no solution satisfying the boundary condition at $u=\frac{1}{2}$. If $E \geqslant 0$, then we can find a suitably behaved solution that vanishes as $u \rightarrow \infty$ and satisfies the boundary condition at $u=\frac{1}{2}$, viz.

$$
\Psi=\left(U U^{\prime}\right)^{1 / 2}\left(J_{1 / 3}(U) J_{-1 / 3}\left(U^{\prime}\right)-J_{1 / 3}\left(U^{\prime}\right) J_{-1 / 3}(U)\right),
$$


where $U=\frac{2}{3} \sqrt{4 E}\left(u-m^{2} / 4 E\right)^{3 / 2}$ and $U^{\prime}=\frac{2}{3} \sqrt{4 E}\left(\frac{1}{2}-m^{2} / 4 E\right)^{3 / 2}$. These solutions are the analog of the scattering states on this manifold subject to the boundary condition we have adopted.

An interesting embedding in pseudo-Euclidean space is given by

$$
X=\sqrt{2 u} v, \quad Y=\sqrt{u}\left(\frac{4}{5} u^{2}-v^{2}+\frac{1}{2}\right), \quad T=\sqrt{u}\left(\frac{4}{5} u^{2}-v^{2}-\frac{1}{2}\right),
$$

for which $d X^{2}+d Y^{2}-d T^{2}=2 u\left(d u^{2}+d v^{2}\right)$. In this case the variables vary over the ranges $-\infty$ $<v<\infty, 0 \leqslant u<\infty$. We could indeed do an analysis of the free Schrödinger equation on this surface and come to a similar conclusion if we imposed the condition that the wave function is zero at $u=0$. However, if we consider the first potential (59) and choose $b_{1}=-\beta^{2}, b_{3}=\frac{1}{4}\left(\frac{1}{4}\right.$ $-\gamma^{2}$ ) for real $\beta$ and $\gamma \geqslant 0$, and if we write the solutions to Schrödinger's equation in the form $\Psi=U(u) V(v)$, then two independent solutions of the separation equation satisfied by $V$ can be taken as

$$
V_{ \pm}=\exp \left(-\frac{1}{2} \beta v^{2}\right) v_{1}^{ \pm \gamma+1 / 2} F_{1}\left(\frac{1}{2}(1 \pm \gamma)-\frac{\mu}{\beta}, 1 \pm \gamma, \beta v^{2}\right)
$$

If we wish to interpret these solutions as being associated with an angle variable which varies in the range $0<v_{0} \leqslant v \leqslant v_{0}+2 \pi$, then we would require the periodic boundary conditions

$$
V\left(v_{0}\right)=V\left(v_{0}+2 \pi\right), \quad V^{\prime}\left(v_{0}\right)=V^{\prime}\left(v_{0}+2 \pi\right) .
$$

The possibility of imposing these boundary conditions depends on whether $v=0$ occurs inside the domain of $v$. If it does not, then the spectrum is determined from the condition

$$
\left.W\left[V_{+}(x)-V_{+}(x+2 \pi), V_{-}(x)-V_{-}(x+2 \pi)\right]\right|_{x=v_{0}}=0 .
$$

If $v=0$ is included, then the same conditions no longer work as this is a regular singular point of the equation. Indeed, if $v_{0}=0$ and we assume $\gamma>\frac{1}{2}$, then we choose the solution $V_{+}$and impose the condition

$$
V_{+}(2 \pi)=0
$$

as $V_{+}(0)$ is already zero. The quantization condition is then determined by

$$
{ }_{1} F_{1}\left(\frac{1}{2}(1+\gamma)-\frac{\mu}{\beta}, 1+\gamma, 4 \beta \pi^{2}\right)=0 .
$$

For the $u$ separation equation the range of variation of the variable $u>\frac{1}{2}$ is clear and $u=\frac{1}{2}$ is not a singularity of the the separation equation. We can accordingly take typical solutions to be

$$
U_{ \pm}(u)=a_{1} D_{\nu}\left(2 \sqrt{\beta}\left(u-\frac{E}{2 \beta^{2}}\right)\right)+a_{2} D_{\nu}\left(-2 \sqrt{\beta}\left(u-\frac{E}{2 \beta^{2}}\right)\right),
$$

where $\nu=(1 / 4 \beta)\left(E^{2} / \beta^{2}+4 b_{2}-\mu\right)-\frac{1}{2}$. To obtain a solution that vanishes as $u \rightarrow \infty$ requires that $a_{2}=0$. The remaining boundary condition becomes

$$
D_{\nu}\left(2 \sqrt{\beta}\left(\frac{1}{2}-\frac{E}{2 \beta^{2}}\right)\right)=0 .
$$

This condition determines the nature of the discrete spectrum. For large eigenvalues the discrete spectrum is given by

$$
E \cong-2 \sqrt{\beta^{3} n}
$$


for suitable large integer $n$.

If we consider the second potential (64), then putting $a_{3}=-\alpha^{2}$ the equation for $V(v)$ has solutions of the form

$$
V=d_{1} D_{\nu}\left(2 \sqrt{\alpha}\left(v-\frac{a_{2}}{2 \alpha^{2}}\right)\right)+d_{2} D_{\nu}\left(-2 \sqrt{\alpha}\left(v-\frac{a_{2}}{2 \alpha^{2}}\right)\right)=d_{1} V_{+}+d_{2} V_{-},
$$

where

$$
\nu=\frac{1}{4 \alpha}\left(4 \mu+\frac{a_{2}^{2}}{\alpha^{2}}\right)-\frac{1}{2} .
$$

As there are no singularities in $v$ in this equation and we can require that $w_{0} \leqslant v \leqslant w_{0}+2 \pi$,

$$
V\left(w_{0}\right)=V\left(w_{0}+2 \pi\right), \quad V^{\prime}\left(w_{0}\right)=V^{\prime}\left(w_{0}+2 \pi\right),
$$

which is equivalent to

$$
\left.W\left[V_{+}(x)-V_{+}(x+2 \pi), V_{-}(x)-V_{-}(x+2 \pi)\right]\right|_{x=w_{0}}=0 .
$$

The solutions for the function $U(u)$ that are well behaved for large $u$ are

$$
U(u)=D_{\rho}\left(2 \sqrt{\alpha}\left(u-\frac{E}{2 \beta^{2}}\right)\right),
$$

where $\rho=(1 / 4 \beta)\left(4 a_{1}-4 \mu+E^{2} / \alpha^{2}\right)-\frac{1}{2}$.

\section{CONCLUSION}

In this article we have examined one of the four spaces of revolution listed by Koenigs. ${ }^{16}$ For the space that we have considered, it has been shown that there are three potentials that can be added to give superintegrable Hamiltonian systems of the type we seek. In each of these cases we have exhibited the various inequivalent ways in which a separation of variables can be achieved for both the classical and quantum equations that result. This is equivalent to determining the various inequivalent ways in which a Hamiltonian can be written in Liouville form (57) for suitable separable coordinates $\alpha, \beta$. In particular, we note that each of the three superintegrable systems we have examined are such that when we write out the classical equation $H=E$ and factor out the denominator we recover a variant of a superintegrable system corresponding to flat space. ${ }^{4}$ This is an example of what is called coupling-constant metamorphosis. ${ }^{20}$ It has been proven in Ref. 12 that all of the superintegrable systems in the plane are such that the bound state energies can be calculated algebraically. In all cases the Hamiltonian lies in the enveloping algebra of $\mathrm{sl}(3, \mathrm{R})$. We conclude that analogous statements apply to the superintegrable systems that we have found.

\section{ACKNOWLEDGMENTS}

One of the authors (P.W.) thanks the Department of Mathematics of the University of Waikato for its hospitality during his visit. The research of P.W. was partly supported by research grants from NSERC of Canada and FCAR du Quebec, and J.K. was supported by the New Zealand Marsden Fund.

\footnotetext{
${ }^{1}$ H. Goldstein, Classical Mechanics (Addison-Wesley, Reading, MA, 1990).

${ }^{2}$ V. I. Arnold, Mathematical Methods of Classical Mechanics (translated by K. Vogtmann and A. Weinstein), Graduate Texts in Mathematics, 60 (Springer-Verlag, New York, 1978).

${ }^{3}$ J. Bertrand, C. R. Acad. Sci. 77, 849 (1873).

${ }^{4}$ I. Fris, V. Mandrosov, J. Smorodinsky, M. Uhlir, and P. Winternitz, Phys. Lett. 16, 354 (1965).
} 
${ }^{5}$ P. Winternitz, J. Smorodinsky, M. Uhlir, and I. Fris, Yad. Fiz. 4, 625 (1966); Sov. J. Nucl. Phys. 4, 132 (1967)

${ }^{6}$ A. Makarov, J. Smorodinsky, K. Valiev, and P. Winternitz, Nuovo Cimento A 52, 1061 (1967).

${ }^{7}$ N. W. Evans, Phys. Rev. A 41, 5666 (1990).

${ }^{8}$ N. W. Evans, J. Math. Phys. 32, 3369 (1991).

${ }^{9}$ E. G. Kalnins, W. Miller, Jr., G. S. Pogosyan, and G. C. Williams, J. Math. Phys. 40, 708 (1999).

${ }^{10}$ E. G. Kalnins, W. Miller, Jr., and G. S. Pogosyan, J. Math. Phys. 37, 6439 (1996).

${ }^{11}$ M. B. Sheftel, P. Tempesta, and P. Winternitz, J. Math. Phys. 42, 659 (2001).

${ }^{12}$ P. Tempesta, A. V. Turbiner, and P. Winternitz, J. Math. Phys. 42, 4248 (2001).

${ }^{13}$ E. G. Kalnins, W. Miller, Jr., and G. S. Pogosyan, J. Phys. A 33, 4105 (2000).

${ }^{14}$ E. G. Kalnins, W. Miller, Jr., and G. S. Pogosyan, J. Phys. A 33, 6791 (2000).

${ }^{15}$ E. G. Kalnins, J. M. Kress, W. Miller, Jr., and G. S. Pogosyan, J. Phys. A 34, 4705 (2001).

${ }^{16}$ G. Koenigs, "Sur les géodésiques a intégrales quadratiques," in Leçons sur la théorie générale des surfaces, edited by G. Darboux (Chelsea, New York, 1972), Vol. 4, pp. 368-404.

${ }^{17}$ G. Darboux, Leçons sur la théorie générale des surfaces, Vols. 1-4 (Chelsea, New York, 1972).

${ }^{18}$ C. Daskaloyannis, J. Math. Phys. 42, 1100 (2001).

${ }^{19}$ P. Létourneau and L. Vinet, Ann. Phys. 243, 144 (1995).

${ }^{20}$ J. Hietarinta, B. Grammaticos, B. Dorizzi, and A. Ramani, Phys. Rev. Lett. 53, 1707 (1984). 